Journal of The Korean Astronomical Society

37: $543 \sim 546,2004$

\title{
A GRADIENT-T SZE
}

\author{
Makoto HatTori and Nobuhiro OKabe \\ Astronomical Institute, Graduate School of Science, Tohoku University, Sendai 980-8578, Japan \\ E-mail: hattori@astr.tohoku.ac.jp, okabe@astr.tohoku.ac.jp
}

\begin{abstract}
The inverse Compton scattering of the cosmic microwave background (CMB) radiation with electrons in the intracluster medium which has a temperature gradient, was examined by the third-order perturbation theory of the Compton scattering. A new type of the spectrum distortion of the CMB was found and named as gradient T Sunyaev-Zel'dovich effect (gradT SZE). The spectrum has an universal shape. There is a zero distortion point, the cross over frequency, at $326 \mathrm{GHz}$. When the hotter region locates closer to an observer, the intensity becomes brighter than the $\mathrm{CMB}$ in the frequency region lower than the cross over frequency and fainter than the CMB in the frequency region higher than the cross over frequency. When the cooler region locates closer to an observer, the distorted part of the spectrum has an opposite sign to the above case. The amplitude of the spectrum distortion does not depend on the electron density and depends on the heat conductivity and the total temperature variation along a line of sight. Therefore, the gradT SZE provides an unique opportunity to measure thermally nonequilibrium electron momentum distribution function in the ICM and combined with the X-ray measurements of the electron temperature distribution provides an opportunity of direct measurement of the heat conductivity in the ICM.
\end{abstract}

Key words : clusters of galaxies - Compton scattering - conduction - magnetic fields - plasma

\section{INTRODUCTION}

The recent dramatic progresses of the X-ray observations have been unveiling that the temperature distribution of the intracluster medium (hereafter ICM) is far from isothermal and the heat conductivity is one of the key parameters which determine the evolution of the ICM(Ikebe et al. 1999, Markevitch et al. 2000, Fabian et al. 2001, Vikhlinin et al. 2001, Markevitch et al. 2003, Zakamska \& Narayan 2003). For example, the global temperature gradient have been found in some cooling core clusters(Kaastra et al. 2004). Zakamska \& Narayan (2003) performed hydrostatic model fitting of the observed temperature and density profile of the ICM assuming the energy balance between the radiative cooling and the conductive heating. They showed that the heat conductivities should be one-third of the Spitzer value in majority of the clusters. Shock waves(Markevitch et al. 2002, Fujita et al. 2004) and cold fronts(Markevitch et al. 2000, Vikhlinin et al. 2001) found in merging clusters are showing the temperature jumps across the discontinuities. The reduction of the heat conductivity across the cold fronts in many order of magnitude are required to maintain the structures(Ettori \& Fabian 2000). The hot bubbles caused by the interaction with the activitiy of the central galaxies have been found in many clusters(McNamara et al. 2000, Fabian et al. 2000, Blanton et al. 2001). The heat conductivities across the surface of the hot bubbles must be reduced in many

Proceedings of The 3rd Korean Astrophysics Workshop on Cosmic Rays and Magnetic Fields in Large Scale Structure order of mangnitudes to maintain the structures. The large scale fluctuation of the temperature distributions have been found in several clusters(Watanabe et al. 1999, Shibata et al. 2001, Markevitch et al. 2003). To maintain the observed temperature fluctuations at least for a dynamical time scale of clusters, the order of magnitude reduction of the heat conductivities are required(Markevitch et al. 2003). Therefore, a direct method for measuring the heat conductivities is desired.

The recent theoretical progresses have been unveiling that a microscopic instability of the plasma which has a temperature gradient(Ramani \& Laval 1978), could play important roles on the suppression of the heat conductivities(Levinson \& Eichler 1992, Hattori \& Umetsu 2000, Okabe \& Hattori 2003) and the origin of cluster magnetic fields(Okabe \& Hattori 2003, Okabe \& Hattori 2004). The heat conduction is the electron thermal energy flow from hot to cold regions. Since it is the third moment of the electron momentum distribution function, the electron momentum distribution function must have deviation from the Maxwell-Boltzmann distribution which is the even function of the momentum. Therefore, the electron momentum distribution function of the plasma with a temperature gradient is described by the sum of the Maxwell-Boltzmann distribution, $g_{\mathrm{m}}(\mathbf{q})=$ $(2 \pi)^{3} n_{e}\left(2 \pi m_{e} k_{B} T_{e}\right)^{-3 / 2} \exp \left(-\frac{q^{2}}{2 m_{e} k_{B} T_{e}}\right)$, and the non Maxwellian part, $\Delta g(\mathbf{q})$, where $\mathbf{q}$ is a momentum of electron, and $n_{e}, T_{e}$ and $m$ are electron number density, temperature and mass, respectively. The heat conduc- 
tive current, $\kappa \nabla T_{e}$, is described by the $\Delta g(\mathbf{q})$ as

$$
\int \frac{d^{3} \mathbf{q}}{(2 \pi)^{3}} \frac{1}{2 m} q^{2} \frac{\mathbf{q}}{m} \Delta g(\mathbf{q})=-f_{\kappa} \kappa_{\mathrm{Sp}} \nabla T_{e}
$$

where $f_{\kappa}$ is the heat conductivity in the Spitzer value unit and $\kappa_{\mathrm{Sp}}$ is the Spitzer heat conductivity. The Spitzer heat conductivity is given by $\kappa_{\mathrm{Sp}}=1.31 \lambda_{e} n_{e} k_{B}$ $\left(\frac{k_{B} T_{e}}{m}\right)^{1 / 2}$ where $\lambda_{e}$ is the electron Coulomb mean free path(Spitzer 1956, Sarazin 1988). The deviation of the electron momentum distribution from the thermal equilibrium distribution(Okabe \& Hattori 2003) in the plasma with a temperature gradient is described by

$$
\Delta g(\mathbf{q})=f_{\kappa} \kappa_{\mathrm{Sp}} \frac{m}{k_{B} n_{e}} \frac{\mathbf{q} \cdot \nabla T_{e}}{q_{t h}^{2} T_{e}}\left(2-\frac{4 q^{2}}{5 q_{t h}^{2}}\right) g_{\mathrm{m}}
$$

where $q_{t h}=\sqrt{2 m_{e} k_{B} \bar{T}_{e}}$ is the electron thermal momentum. The plasma instability is seeded by this local deviation of the electron momentum distribution from the thermal equilibrium distribution. Therefore, the establishing the functional form of the non-equilibrium momentum distribution function with the observation is crucial for confirming the above mentioned theoretical models on the suppression of the heat conductivities and generation of the magnetic fields. Details of the derivations and the application to clusters are described in Hattori and Okabe (2004).

In this paper, we examined a spectrum distortion of the cosmic microwave background (hereafter CMB) due to the inverse Compton scattering with electrons which support heat conduction, is examined by solving the Boltzmann equation for Compton scattering to thirdorder in the electron momentum. The result provides a direct method for measuring the heat conductivities and the functional form of the electron momentum distribution functions in the plasma with a temperature gradient.

\section{THE BOLTZMANN EQUATION FOR THIRD-ORDER COMPTON SCATTER- ING}

The spectrum distortion of the cosmic microwave background (CMB) due to the Compton scattering from electrons with momentum distribution function of $g(\mathbf{q})$ is given by the Boltzmann equation( $\mathrm{Hu}$ et al. 1994) as

$$
\begin{aligned}
\frac{D f(\mathbf{x}, \mathbf{p})}{c D t} & =\frac{1}{2 E(\mathbf{p})} \int D \mathbf{q} D \mathbf{q}^{\prime} D \mathbf{p}^{\prime}(2 \pi)^{4} \\
& \times \delta^{(4)}\left(\vec{p}+\vec{q}-\vec{p}^{\prime}-\vec{q}^{\prime}\right)|M|^{2} \\
& \times\left\{g\left(\mathbf{x}, \mathbf{q}^{\prime}\right) f\left(\mathbf{x}, \mathbf{p}^{\prime}\right)[1+f(\mathbf{x}, \mathbf{p})]\right. \\
& \left.-g(\mathbf{x}, \mathbf{q}) f(\mathbf{x}, \mathbf{p})\left[1+f\left(\mathbf{x}, \dot{\mathbf{p}}^{\prime}\right)\right]\right\}
\end{aligned}
$$

where $f(\mathbf{x}, \mathbf{p})$ is the photon occupation number with a three-momentum $\mathbf{p}, \mathbf{p}$ and $\mathbf{p}^{\prime}$ are the three-momentum for photons of four-momentun $\vec{p}$ and $\vec{p}^{\prime}$, $\mathbf{q}$ and $\mathbf{q}^{\prime}$ are the three-momentum for electrons of four-momentun $\vec{q}$ and $\vec{q}^{f}, E(\mathbf{p})$ is the energy of the photon with a momentum p, $|M|^{2}$ is the Lorentz-invariant matrix element for Compton scattering( $\mathrm{Hu}$ et al. 1994), and

$$
D \mathbf{q}=\frac{d^{3} \mathbf{q}}{(2 \pi)^{3} 2 E(\mathbf{q})}
$$

is the Lorentz-invariant phase space volume element of electrons with an energy of $E(\mathbf{q})$ and a momentum q. The right hand side of the Boltzmann equation is the collision term. The first and the second terms are the contributions from scattering into and out of the momentum state $\mathbf{p}$ including the stimulated emission effects, respectively.

When the electron velocity distribution function is the Maxwell-Boltzmann distribution and the thermal energy contained in electron system is much less than the electron rest mass energy, by expanding the collision term of the Boltzmann equation for the initially isotropically distributed photons up to the second-order in electron momentum, the Kompaneets equation, describing the evolution of the photon distribution function due to the Compton scattering, is deduced(Hu et al. 1994, Kompaneets 1957). The spectrum distortion of the CMB due to the Compton scattering from thermal electrons contained in the clusters of galaxies are obtained by solving the Kompaneets equation under the conditions that the probability of the multiple scattering of a photon is negligibly small and the electron temperature is much higher than the temperature of the CMB. This effect is known as the thermal SunyaevZel'dovich effect (hereafter thermal SZE) (Sunyaev \& Zel'dovich 1972).

The spectrum distortion due to the plasma with a temperature gradient is deduced by the following way. In the plasma which has a temperature gradient, the electron momentum distribution function is described as $g(\mathbf{q})=g_{\mathrm{m}}(\mathbf{q})+\Delta g(\mathbf{q})$. Since the multiple scattering effect of a photon is negligible in the clusters of galaxies, the effects of the spectrum distortion of the CMB due to the Compton scattering from electrons with $g_{\mathrm{m}}(\mathbf{q})$ and electrons with $\Delta g(\mathbf{q})$ are decoupled. Therefore, each effects can be evaluated separately and the total spectrum distortion is given by the superposition of each results. Hereafter, the SZE due to electrons with $\Delta g(\mathbf{q})$ is referred to gradT SZE. Since the first non-zero moment of the $\Delta g(\mathbf{q})$ appears from the third-order moment, to evaluate the effect of the $\Delta g(\mathbf{q})$ on the CMB spectrum distortion the third order expansion in electron momentum of the collision term of the Boltzmann equation is required. A final result of third-order expansion is reduced to

$$
\begin{aligned}
\frac{D f(\mathbf{x}, \mathbf{p})}{c D t}= & 3 f_{\kappa} \kappa_{\mathrm{Sp}} \frac{\sigma_{\mathrm{T}}}{m c^{3}} \mathbf{n} \cdot \nabla T_{e}\left[x f^{\prime}(\mathbf{x}, \mathbf{p})\right. \\
& \left.+\frac{94}{75} x^{2} f^{\prime \prime}(\mathbf{x}, \mathbf{p})+\frac{14}{75} x^{3} f^{\prime \prime \prime}(\mathbf{x}, \mathbf{p})\right],(3)
\end{aligned}
$$

where $x=\frac{h \nu}{k_{B} T_{\gamma}}, h$ is the Planck constant, $c$ is a speed 
of light, $\nu$ is the frequency of the CMB photon, $T_{\gamma}$ is the temperature of the $\mathrm{CMB}, \sigma_{\mathrm{T}}$ is the Thomson cross section and $\mathbf{n} \equiv \frac{\mathbf{p}}{p}$ is the direction cosine of the observed photon, respectively.

\section{THE SPECTRUM OF THE GRADIENT- T SZE}

The spectrum of the gradT SZE is obtained by inserting the Planck function in the right hand side of the above obtained third-order Boltzmann equation and integrating over line of sight. The frequency dependent amplitude of the distortion expressed in temperature is obtained as

$$
\begin{aligned}
\Delta T_{\nu}^{\nabla T}= & \frac{12 \sigma_{\mathrm{T}}}{m c^{3}} T_{\gamma} \int d \ell f_{\kappa} \kappa_{\mathrm{Sp}} \mathbf{n} \cdot \nabla T_{e} \frac{1}{x^{2}} \Delta^{\nabla T}(x \nmid 4) \\
\Delta^{\nabla T}(x) \equiv & -\frac{x^{4} \mathrm{e}^{x}}{\left(\mathrm{e}^{x}-1\right)^{2}}\left[1-\frac{94}{75} x \operatorname{coth} \frac{x}{2}\right. \\
& \left.+\frac{14}{75} x^{2}\left(\operatorname{coth}^{2} \frac{x}{2}+\frac{1}{2 \sinh ^{2} \frac{x}{2}}\right)\right],
\end{aligned}
$$

where $d \ell$ is the line of sight integral. Figure 1 shows the frequency dependence of the kernel function $\Delta^{\nabla T}(x)$ and compares it with the thermal and kinetic SZE(Hu et al. 1994, Birkinshaw 1999). The spectrum shows distinct characteristics compared with others. It has a finite value at $218 \mathrm{GHz}$ where the thermal SZE is always zero. The location of maxima, zero, and minima are independent of $T_{e}$ and they are at $x_{\max }=$ $3.44(\nu=195 \mathrm{GHz}), x_{\text {zero }}=5.75 ;(\nu=326 \mathrm{GHz})$, and $x_{\min }=8.36 ;(\nu=437 \mathrm{GHz})$, respectively. Since the amplitude of the spectral distortion is proportional to the inner product of the $\mathbf{n}$ with $\nabla T_{e}$, the spectrum of the $\operatorname{grad} T$ SZE has the same shape as the kernel function when the hotter region locates closer to the observer but it has the opposite sign to the kernel function when the cooler region locates closer to the observer. Therefore, a spherically symmetric system, for example, does not show the spectrum distortion due to the gradT SZE. When the line of sight is exactly perpendicular to the temperature gradient, the gradT SZE is zero.

The amplitude of the spectrum distortion due to the $\operatorname{gradT}$ SZE at the peak frequency is evaluated by the following way. The line of sight integral in the right hand side of the equation is reduced to $T_{e}^{7 / 2}$ and it is independent from the electron density. This is because the heat conductivity does not depend on the elctron density explicitly unless it is saturated(Cowie \& McKee 1977). As decreasing the electron density the Coulomb mean free path becomes longer. In the region where the electron density is lower than $10^{-5} \mathrm{~cm}^{-3}$ with $k_{B} T_{e}=10 \mathrm{keV}$, the mean free path is longer than $2 \mathrm{Mpc}$ (Sarazin 1988). For the typical rich cluster, the electron density gets lower than $10^{-5} \mathrm{~cm}^{-3}$ in the radius larger than $2 \mathrm{Mpc}$. Therefore, in such a cluster out skirt any temperature gradient scale is longer than the electron Coulomb mean free path and the heat conduction is saturated. As modeled by the Cowie and

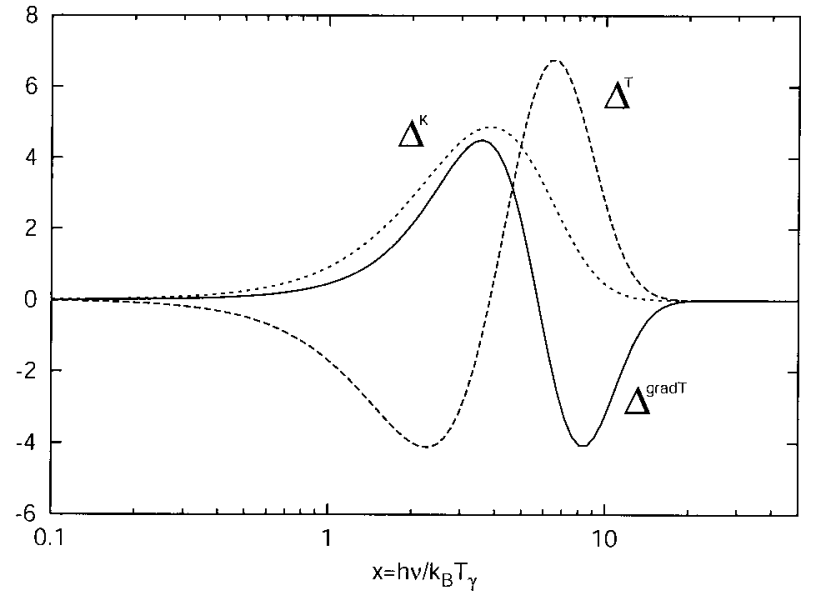

Fig. 1.- Comparison of the CMB spectrum distortion due to the gradT SZE with the thermal SZE and the kinetic SZE. The solid line shows the kernel function of the gradT SZE. Dashed line and dotted line are the kernel function of the thermal SZE, $\Delta^{T}(x)=x^{4} e^{x} /\left(e^{x}-1\right)^{2}(x \operatorname{coth}(x / 2)-4)$, and the kinetic SZE, $\Delta^{K}(x)=x^{4} e^{x} /\left(e^{x}-1\right)^{2}$, respectively(Birkinshaw 1999). A horizontal axis is the frequency of the CMB photons normalized by its temperature. A vertical axis is described in arbitrary unit.

Mackee (1977), the saturated heat conductivity could be evalued by the collisionless diffusion of the hot electrons with the Maxwellian velocity distribution. We also assume that the electron velocity distribution is the Maxwellian in the region where the Coulomb mean free path is longer than the temperature gradient scale since the deviation of the velocity distribution function from the thermal equilibrium form is hard to be established without any collision between particles. Therefore, $\Delta g=0$ in the region where the heat conduction is saturated and no gradient-T SZE is generated in this region. Suppose that $\Delta g$ disappears suddenly at the points where the electron Coulomb mean free path becomes as long as the temperature gradient scale. The temperature of these points are denoted by $T_{e 2}$ and $T_{e 1}$, respectively for the region closer side to the observer and far side to the observer along the line-of-sight. The amplitude of the gradT SZE is described as

$$
\begin{aligned}
\Delta T_{\nu}^{\nabla T} & =0.8\left(\frac{f_{\kappa}}{1.0}\right) \\
& \times\left[\left(\frac{k_{B} T_{e 2}}{10 \mathrm{keV}}\right)^{3.5}-\left(\frac{k_{B} T_{e 1}}{5 \mathrm{keV}}\right)^{3.5}\right] \mu \mathrm{K} .
\end{aligned}
$$

The result shows that the amplitude of the gradT SZE is proportional to the heat conductivity. However, to extract the heat conductivity from the observed amplitude of the gradT SZE, the electron temperature in the cluster out skirt of the both side of the line-of-sight have to be measured and it is practically difficult. The expected amplitude is two order of magnitude less than the thermal SZE and an order of magnitude less than 
the kinetic SZE but order of magnitude larger than the gravitational scattering effect. To distinguish the gradT SZE signal from other SZE signal, measurement of the spectrum by broad band or multi-band observations are required.

\section{DISCUSSION}

We have shown that the inverse Compton scattering of the CMB photon with electrons in the ICM which has a temperature gradient, results in a new type of the spectrum distortion of the CMB by the third-order perturbation theory of the Compton scattering. The spectrum has an universal shape. There is a zero distortion point at $326 \mathrm{GHz}$. When the hotter region locates closer to the observer, the intensity becomes brighter and fainter than the CMB spectrum in the frequency regions lower and higher than $326 \mathrm{GHz}$, respectively. When the cooler region locates closer to observer, the distorted part of the spectrum has an opposite sign to the above case. The amplitude of the spectrum distortion is proportional to the heat conductivity and the temperature at the points where the heat conduction becomes saturated both of the close and far sides to the observer along the line-of-sight, and does not depend on the electron density. Therefore, the effect is distinguishable from other SZE effects by broad band or multi-bands observations of the SZE. It provides an unique opportunity of measuring the non-Maxweillian part of the electron momentum distribution function when the finite heat conduction exists and of direct measurement of the heat conductivity in cluster hot gas.

The gradT SZE does not cause any polarization since the quadrapole moment of the CMB intensity distribution averraged over all the electron velocity is zero. The linear polarization of the Compton scattered photons is generated by the systematic transverse motion of the electron system since it leads a finite quadrapole moment of the CMB intensity distribution in the frame moving with the same velocity as the transverse motion of the electron system(Sunyaev \& Zel'dovich 1980). As explained in the previous section and appendix, the first and second moments of the eletron velocity on $\Delta g(\mathbf{q})$ are zero(Okabe \& Hattori 2003). Therefore, the $\Delta g(\mathbf{q})$ does not generate a quadrapole moment of the CMB intensity distribution.

The expected signal of the gradT SZE in real clusters are summarized as follows. When the line-of-sight acrosses the cluster merging shock, the amplitude of $\sim 10 \mu \mathrm{K}$ is expected at most. This could be detectable by ALMA/ACA. To distinguish the gradT SZE signal from other SZE signals, measurement of the spectrum by broad band or multi-band observations are required. The expected signals in other situations are smaller than $\mu \mathrm{K}$ and are unfortunately very small.

\section{ACKNOWLEDGEMENTS}

This work is supported by a Grant-in-Aid for the 21st Century COE Program "Exploring New Science by Bridging Particle-Matter Hierarchy" in Tohoku University, funded by the Ministry of Education, Science, Sports and Culture of Japan and a Grant-in-Aid No.16204010 funded by Japan Society for the Promotion of Science.

\section{REFERENCES}

Blanton, E. L., Sarazin, C. L., McNamara, B. R., Wise, M. W. 2001, ApJ, 558, L15

Birkinshaw, M. 1999, Phys. Rep., 310, 97

Cowie, L. L. \& McKee,C.F. 1977, ApJ, 211, 135

Ettori, S. \& Fabian, A. C. 2000, MNRAS, 317, L57

Fabian, A. C. et al. 2000, MNRAS, 318, L65

Fabian, A. C. et al. 2001, MNRAS, 321, L33

Fujita, Y. et al. 2004, ApJ, in press

Hattori, M., Kneib, J.-P. \& Makino, N. 1999, Prog. Theor. Phys. Suppl., 133, 1

Hattori, M. \& Umetsu, K. 2000, ApJ, 533, 84

Hattori, M. \& Okabe, N. 2004, submitted to ApJ.

Hu,W., Scott, D. \& Silk, J. 1994, Phys. Rev. D, 49, 648

Ikebe, Y. et al. 1999, ApJ, 525, 58

Kaastra, J. S. et al. 2004, A\&A, 413, 415

Kitayama, T. et al. 2004, PASJ in press

Kompaneets, A. S. 1957, Sov. Phys. JETP, 4, 730

Levinson, A. \& Eichler, D. 1992, ApJ, 387, 212

Markevitch, M. et al. 2000, ApJ, 541, 542

Markevitch, M. et al. 2002, ApJ, 567, L27

Markevitch, M. et al. 2003, ApJ, 586, L19

McNamara, B. R. et al. 2000, ApJ, 534, L135

Okabe, N. \& Hattori, M. 2003, ApJ, 599, 964

Okabe, N. \& Hattori, M. 2004, ApJ, submitted

Ramani,A . \& Laval, G. 1978, Phys. Fluids, 21, 980

Sarazin, C. L., X-ray emission from Clusters of Galaxies (Cambridge: Cambridge Univ. Press, 1988)

Shibata, R., et al. 2001, ApJ, 549, 228

Spitzer, L. Jr. 1956, Physics of Fully Tonized Gases (New York, Interscience)

Sunyaev, R. A. \& Zel'dovich, Ya. B. 1972, Com. Ap. Sp. Phys., 4, 173

Sunyaev, R. A. \& Zel'dovich, Ya. B. 1980, MNRAS, 190, 413

Takizawa, M. 1999, ApJ, 520, 514

Vikhlinin, A., Markevitch, M. \& Murray, S. S. 2001, ApJ, 549, L47

Watanabe, M., et al. 1999, ApJ, bf 527, 80

Zakamska, N. \& Narayan, R. 2003, ApJ, 582, 162 